\title{
New Constant Mean Curvature Surfaces
}

\author{
Martin Kilian, Ian Mclntosh, and Nicholas Schmitt
}

\section{CONTENTS}

1. Introduction

2. The DPW Construction

3. CMC Cylinders

4. Concluding Remarks

5. Appendix by Nicholas Schmitt: K-noids

Electronic Availability

Acknowledgments

References

AMS Subject Classifcation: 53A10
We use the Dorfmeister-Pedit-Wu construction to present three new classes of immersed CMC cylinders, each of which includes surfaces with umbilics. The first class consists of cylinders with one end asymptotic to a Delaunay surface. The second class presents surfaces with a closed planar geodesic. In the third class each surface has a closed curve of points with a common tangent plane. An appendix, by the third author, describes the DPW potentials that appear to give CMC punctured spheres with $\mathrm{k}$ Delaunay ends ( $\mathrm{k}$-noids): the evidence is experimental at present. These can have both unduloidal and nodoidal ends.

\section{INTRODUCTION}

Dorfmeister, Pedit and Wu presented in [Dorfmeister et al. 1998] a method by which all immersed CMC surfaces can, in principle, be constructed. The construction is based on the observation that the Gauss map of every CMC surface is harmonic and every harmonic map from a surface $D$ to $S^{2}$ is the projection of a horizontal holomorphic map from its universal cover $\tilde{D}$ into a certain loop group. Thus the data for the DPW method is a holomorphic 1form with values in a certain loop algebra: this is called a holomorphic potential. One of the difficulties in using this method to construct new surfaces is that if the potential actually lives on $D$ it need not follow that it produces an immersion of $D$. We usually only obtain an immersion of $\tilde{D}$ : this is the closing (or monodromy) problem. Therefore part of the purpose here is to present some examples of solving the closing problem in the simplest case, where $D=\mathbb{C}^{*}$.

The simplest known examples of CMC cylinders are the Delaunay surfaces, which are characterized by being cylinders of revolution (this includes the standard cylinder). From [Korevaar et al. 1989] one knows that if a CMC cylinder is complete and properly embedded then it must be a Delaunay surface. Also, every properly embedded annular end must be 
a Delaunay end (i.e., asymptotic to a Delaunay surface) even if the surface is not embedded [Korevaar et al. 1989]. For example, the 'bubbletons' studied in [Sterling and Wente 1993] are immersed cylinders with no umbilics and both ends asymptotic to the standard cylinder. The examples we will present include cylinders which have one Delaunay end and any number of umbilics.

In fact we present three new classes of CMC cylinders. The first class includes surfaces which are best thought of as a Smyth surface [Smyth 1993; Dorfmeister et al. 1998] with the head replaced by a Delaunay end. Given the results of [Timmreck et al. 1994] on Smyth surfaces we conjecture that these new examples are complete and proper immersions. Indeed these surfaces come in one-parameter families each of which includes a Smyth surface (with the umbilic removed) as a degenerate limit, in the same way that the Delaunay surfaces are a one-parameter family containing the sphere (with two points removed) as a degenerate limit. The next class consists of CMC cylinders which contain a closed planar geodesic. The third class presents cylinders each of which admits a closed curve of points with common tangent plane.

Although it is very easy to read off the Hopf differential from the potential, it is usually unclear how the geometry of the surface is encoded in the potential. For example, there is as yet no understanding of the conditions on a potential which ensure that the surface is either proper, complete or embedded. The main obstacle in understanding the passage from the potential to the surface is a loop group factorization (the Iwasawa decomposition). This led us to build a numerical package that would compute this factorization and produce images of the surface: the approach is described below. The result is a computer laboratory, called dpwlab, written by the third author. Other attempts have been made to implement the DPW method numerically (see [Lerner and Sterling 1995], for example), but they find the Iwasawa decomposition by first turning it into a Riemann-Hilbert problem (Birkhoff factorization). The dpwlab directly computes the Iwasawa decomposition according to the theory described in [Pressley and Segal 1986].

An appendix, by the third author, introduces a class of DPW potentials that appear to give the tri- unduloid surfaces classified in [Große-Brauckmann et al. 1999]. In fact the experiments predict that there is another family of 3-punctured spheres, with two unduloidal ends and one nodoidal end. A generalization of the balancing formulae [Kusner 1991] applies to these latter surfaces even though they are no longer almost embedded. Moreover, these ideas are extended to produce a family of DPW potentials which will, it is conjectured, produce symmetric $k$ punctured spheres with equal asymptotic necksizes: the so-called equilateral $k$-noids. The experiments support this conjecture.

\section{THE DPW CONSTRUCTION}

Before stating the DPW recipe, we introduce the ingredients. For $G \subset \operatorname{gl}(2, \mathbb{C})$, denote the analytic maps of the unit circle $S^{1}$ with values in $G$ by $\Lambda G$ and define the twisted loops by

$$
\Lambda^{\sigma} G=\left\{g \in \Lambda G: g(-\lambda)=\sigma g(\lambda) \sigma^{-1}\right\},
$$

where $\sigma=\left(\begin{array}{rr}1 & 0 \\ 0 & -1\end{array}\right)$. Furthermore, define

$$
\begin{aligned}
\Lambda_{+}^{\sigma}=\left\{g \in \Lambda^{\sigma} \mathrm{SL}(2, \mathbb{C})\right. & : g(\lambda)=\sum_{k \geq 0} g_{k} \lambda^{k}, \\
g_{0} & \left.=\left(\begin{array}{cc}
a & b \\
0 & a^{-1}
\end{array}\right) \text { for } a \in \mathbb{R}^{+}\right\} .
\end{aligned}
$$

The principal tool in the DPW method is the loop group Iwasawa decomposition: any $\Phi \in \Lambda^{\sigma} \mathrm{SL}(2, \mathbb{C})$ factorizes uniquely into a product $\Phi=F b$ where $F \in \Lambda^{\sigma} \mathrm{SU}(2)$ and $b \in \Lambda_{+}^{\sigma}$.

Now let us recall the DPW construction. Let $D$ be a Riemann surface and $\tilde{D}$ its universal cover. Denote by $\Omega_{D}^{1,0}$ the holomorphic 1 -forms on $D$. Also define

$$
\begin{aligned}
& \Lambda_{-1, \infty}^{\sigma} \operatorname{sl}(2, \mathbb{C}) \\
& \quad=\left\{g \in \Lambda^{\sigma} \operatorname{sl}(2, \mathbb{C}): g(\lambda)=\sum_{k=-1}^{\infty} g_{k} \lambda^{k}\right\} .
\end{aligned}
$$

The following steps (see [Dorfmeister et al. 1998]) give an $S^{1}$ family, called the associated family, of immersions (possibly with branch points) $f_{\lambda}: \tilde{D} \rightarrow$ $\mathbb{R}^{3} \cong \mathrm{su}(2)$ with constant mean curvature $H$.

1. Let $\xi_{\lambda}=\sum_{k=-1}^{\infty} \xi_{k}(z) \lambda^{k} \in \Omega_{D}^{1,0} \otimes \Lambda_{-1, \infty}^{\sigma} \operatorname{sl}(2, \mathbb{C})$ and solve the initial value problem

$$
d \Phi_{\lambda}=\Phi_{\lambda} \xi_{\lambda}, \quad \Phi_{\lambda}\left(w_{0}\right)=g
$$

where $w_{0} \in \tilde{D}$ and $g \in \Lambda^{\sigma} \operatorname{SL}(2, \mathbb{C})$. Then $\Phi_{\lambda}$ is defined on $\tilde{D}$. 
2. Apply the Iwasawa decomposition to $\Phi_{\lambda}$ pointwise on $\tilde{D}$ to obtain $\Phi_{\lambda}=F_{\lambda} b_{\lambda}$.

3. The Sym-Bobenko formula yields

$$
f_{\lambda}=-\frac{1}{H}\left(i \lambda \frac{\partial F_{\lambda}}{\partial \lambda} F_{\lambda}^{-1}+\frac{1}{2} F_{\lambda} e_{1} F_{\lambda}^{-1}\right),
$$

where $e_{1}=i \sigma$.

We call the 1-form $\xi_{\lambda}$ the holomorphic potential and $\Phi_{\lambda}$ the extended holomorphic frame. The unitary factor $F_{\lambda}$ is called the extended unitary frame. Our principal interest in this paper is to construct examples where $D=\mathbb{C}^{*}$ and provide sufficient conditions to ensure that the resultant map $f_{\lambda}$ is also defined on $\mathbb{C}^{*}$ for $\lambda=1$.

\section{A. Properties}

We list here a number of properties of the construction which will be relevant for our surfaces.

Group actions. Notice that the surface depends on the data $\xi_{\lambda}, g, w_{0}$. It is clear from the construction that the infinite dimensional group $\mathcal{G}$ of holomorphic maps $\gamma: \tilde{D} \rightarrow \Lambda_{+}^{\sigma}$ with $\gamma\left(w_{0}\right)=I$ acts by gauge transformation on the fibers of the map $\left(\xi_{\lambda}, g, w_{0}\right) \mapsto f_{\lambda}$, since the map $\Phi_{\lambda} \rightarrow \Phi_{\lambda} \gamma$ leaves the surface unchanged. In fact one can always gauge away the diagonal terms of $\xi_{\lambda}$. Another group action is the left action of $\Lambda^{\sigma} \mathrm{SL}(2, \mathbb{C})$ on the initial condition, $g \mapsto h g$ for $h \in \Lambda^{\sigma} \operatorname{SL}(2, \mathbb{C})$, which is called the dressing action; compare [Burstall and Pedit 1995; Dorfmeister and Wu 1993]. It is not hard to see from the Sym-Bobenko formula that the dressing action of the subgroup $\Lambda^{\sigma} \mathrm{SU}(2)$ can only result in Euclidean motions of the surface, therefore it is more usual to think of the dressing action as being by $\Lambda_{+}^{\sigma}$.

Metric and Hopf differential [Dorfmeister et al. 1998]. We may write

$$
F_{\lambda}^{-1} d F_{\lambda}=\alpha_{1}^{(1,0)} \lambda^{-1}+\alpha_{0}+\alpha_{1}^{(0,1)} \lambda,
$$

where $\alpha_{0}, \alpha_{1}$ are 1 -forms on the CMC surface. A simple calculation shows that

$$
\alpha_{1}^{(1,0)}=b_{0} \xi_{-1} b_{0}^{-1} .
$$

Further, if we write

$$
\xi_{-1}=\left(\begin{array}{cc}
0 & a_{1} \\
a_{2} & 0
\end{array}\right) \text { and } b_{0}=\left(\begin{array}{cc}
r & 0 \\
0 & r^{-1}
\end{array}\right)
$$

for $a_{1}, a_{2} \in \Omega_{D}^{1,0}$ and $r: \tilde{D} \rightarrow \mathbb{R}^{+}$, then it can be shown that $f_{1}$ has metric $4 r^{4}\left|a_{1}\right|^{2}$ and Hopf differential $Q=-\frac{1}{2} a_{1} a_{2}$. It follows that $f_{1}$ has branch points at the zeroes of $a_{1}$ and umbilics at the zeroes of $a_{2}$. When $f_{1}$ is unbranched (and away from umbilics) the metric can be written as $e^{u}|d w|^{2}$ for a local conformal coordinate $w$ on $\tilde{D}$ and we have

$$
F_{\lambda}^{-1} \frac{\partial F_{\lambda}}{\partial w}=\left(\begin{array}{cc}
-\frac{1}{4} u_{w} & -\frac{1}{2} H e^{u / 2} \lambda^{-1} \\
\bar{Q} e^{-u / 2} \lambda^{-1} & \frac{1}{4} u_{w}
\end{array}\right)
$$

Symmetries. We cannot usually expect the symmetries of the potential to be passed on to the CMC immersion because they might not survive the combination of integration and factorization. However, there are two situations which occur in our examples where symmetries will appear in the CMC surface.

A. Suppose $\rho \in \operatorname{Aut}(\tilde{D})$ is an automorphism with $w_{0}$ as a fixed point and $\rho^{*} \xi=\mu(\xi)$ where $\mu \in$ $\operatorname{Aut}(\mathrm{sl}(2, \mathbb{C}))$ preserves the subalgebra $\mathrm{su}(2)$. Since the base point $w_{0}$ is fixed, the solution to $(2-1)$ will satisfy $\rho^{*} \Phi_{\lambda}=\mu\left(\Phi_{\lambda}\right)$. Now, since $\mu$ (or rather, its lift to the group) preserves $\mathrm{SU}(2)$, we have $\rho^{*} F_{\lambda}=$ $\mu\left(F_{\lambda}\right)$ by uniqueness of the Iwasawa decomposition. Thus, by the Sym-Bobenko formula, $\rho^{*} f_{\lambda}=\mu\left(f_{\lambda}\right)$. We will produce examples of this below, where $\rho$ is a real involution on $\mathbb{C}$ and $\mu$ is either the identity or $\mu(\xi)=-\bar{\xi}^{t}$. These produce reflection symmetries of the immersion.

B. Suppose that $\nu \in \operatorname{Aut}(\tilde{D})$ induces a finite order automorphism on $D$, of order $n$, and that these conditions hold:

(a) $\nu^{*} \xi_{\lambda}=\xi_{\lambda}$.

(b) $h=\Phi_{\lambda}\left(\nu\left(w_{0}\right)\right) \Phi_{\lambda}\left(w_{0}\right)^{-1}$ belongs to $\Lambda^{\sigma} \mathrm{SU}(2)$.

(c) $f_{1}$ is an immersion of $D$ itself.

Then we may conclude that $f_{1}$ has an $n$-fold rotational symmetry by the following argument. By (b) $\nu^{*} \Phi_{\lambda}=h \Phi_{\lambda}$, so by uniqueness of the Iwasawa decomposition $\nu^{*} F_{\lambda}=h F_{\lambda}$. Therefore $\nu^{*} f_{1}=R\left(f_{1}\right)$ where $R$ is a Euclidean motion. But $R$ must have order $n$, therefore it is a rotation. In the examples below we will have $D=\mathbb{C}^{*}$ and $\nu$ will induce a rotation on $\mathbb{C}^{*}$.

\section{B. Known Examples}

Two classes of known examples will turn out to play an important role in understanding the new surfaces we will examine later: 
Example 1. Recall from [Dorfmeister et al. 1998] that potentials of the form

$$
\xi_{\lambda}=\lambda^{-1}\left(\begin{array}{cc}
0 & 1 \\
p(z) & 0
\end{array}\right) d z
$$

where $p(z)=k z^{m}$, for $m \in \mathbb{N} \cup\{0\}$ and any constant $k$, give the Smyth surfaces [1993]. These surfaces are characterized as CMC planes which possess an intrinsic isometric $S^{1}$-action (with a fixed point). If we think of these as singly punctured (topological) spheres, they have one end with $m+2$ 'legs' and an $(m+2)$-fold rotational symmetry. We must beware of some degenerate cases: taking $p \equiv 0$ yields the round sphere while $p \equiv \pm 1$ gives the standard cylinder. The asymptotics of these ends have been quite thoroughly studied. In [Timmreck et al. 1994] it was shown that these surfaces are proper immersions and that for $\varphi_{n}=\pi n /(m+2), n=0, \ldots, 2 m+3$, there are polar coordinate rays $t \mapsto f\left(t e^{i \varphi_{n}}\right)$ which are planar geodesics. The curvature of these, in the limit as $t \rightarrow \infty$, tends to 0 for $n$ even and 1 for $n$ odd. The legs develop around those lines with $n$ even, along which the distance from the origin grows fastest. The angle between the legs depends upon the coefficient $k$. Further, it was shown in [Bobenko 1991] that the surface is bounded by a cone.

More general surfaces can be obtained by allowing $p(z)$ to be any polynomial [Dorfmeister et al. 1998]. The resultant surfaces have $m+2$ legs, where $m=\operatorname{deg}(p)$, each of which looks like a Smyth surface leg. To the best of our knowledge, there has

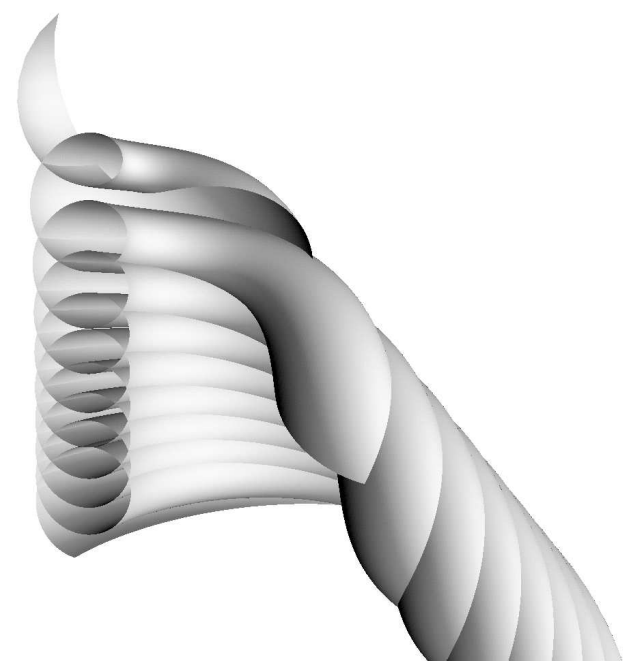

FIGURE 1. Sector of a Smyth surface bounded by a nodoidal planar geodesic. not been any work which describes the strength of this resemblance. Of course, these surfaces need not possess either intrinsic or extrinsic symmetries.

Example 2. All the Delaunay surfaces can be obtained with the family of potentials

$$
\xi^{\text {Del }}=A_{\lambda} \frac{d z}{z}
$$

where

$$
A_{\lambda}=A_{\lambda}(a, b, c)=\left(\begin{array}{cc}
c & a \lambda^{-1}+\bar{b} \lambda \\
b \lambda^{-1}+\bar{a} \lambda & -c
\end{array}\right) .
$$

Here $z$ is a coordinate on $\mathbb{C}^{*}$ and we use as the universal cover the map $\mathbb{C} \rightarrow \mathbb{C}^{*}$ taking $w$ to $z=$ $\exp (i w)$. The conditions $a b \in \mathbb{R}, c \in \mathbb{R}$ with

$$
|a+\bar{b}|^{2}+c^{2}=\frac{1}{4}
$$

ensure that the map has period $2 \pi$ (here, $\lambda$ is evaluated at 1 in the Sym-Bobenko formula). The signed neck and bulge radii of Delaunay surface with this potential (respectively $r_{-}$and $r_{+}$) are

$$
r_{ \pm}=\frac{1 \pm \sqrt{1-16 a b}}{2 H} .
$$

The potential $\xi^{\text {Del }}$ can be normalized by conjugation by a diagonal element of $\operatorname{su}(2)$ so that $a, b \in \mathbb{R}$. The parameter $c$, although not strictly necessary, is sometimes useful. Its geometric effect is to introduce a phase shift along the profile curve of the Delaunay surface. Unduloids and nodoids are obtained when $a b>0$ and $a b<0$ respectively. The limiting case $a b=0$ yields a sphere with two points removed. In fact the gauge transformation

$$
\xi_{\lambda} \mapsto \gamma^{-1} \xi_{\lambda} \gamma+\gamma^{-1} d \gamma
$$

with

$$
\gamma=\left(\begin{array}{cc}
\sqrt{z} & 0 \\
-\lambda \sqrt{z} & 1 / \sqrt{z}
\end{array}\right),
$$

transforms the potential $(2-2)$ (on $\mathbb{C}^{*}$ ) with $p \equiv 0$ into the potential (2-3) with $a=1, b=0, c=0$. This gauge transformation will be useful later on.

Below we will use $\Phi_{\lambda}^{\text {Del }}$ and $F_{\lambda}^{\text {Del }}$ to denote respectively the holomorphic and unitary extended frames for the potential $\xi_{\lambda}^{\text {Del }}$ with $\Phi_{\lambda}^{\text {Del }}(0)=I$. In particular, notice that $\Phi_{\lambda}^{\mathrm{Del}}=\exp \left(i w A_{\lambda}\right)$.

Remark. The Delaunay potentials fit into the following more general context. By [Burstall and Pedit 1994, Theorem 4.3], each CMC surface with doubly 
periodic Gauss map can be obtained from a holomorphic potential $\xi_{\lambda}$ on $\mathbb{C}$ which is constant along the plane and with $g=I, w_{0}=0$ in $(2-1)$. Recall from [Bobenko 1991] that each such surface is partially characterized by its spectral curve, which is a Riemann surface with equation of the form

$$
\mu^{2}=\zeta \prod_{j=1}^{g}\left(\zeta-c_{j}\right)\left(\zeta-\bar{c}_{j}^{-1}\right)
$$

with $0 \leq\left|c_{j}\right| \leq 1$. There is a $(g-2)$-parameter family of CMC surfaces with the same spectral curve: for $g=1$ there is one surface for each spectral curve and this surface is a Delaunay surface. It can be shown (we omit the proof here) that one of the surfaces with the spectral curve above can be obtained by taking

$$
\xi_{\lambda}=\lambda^{2 g-1}\left(\begin{array}{cc}
0 & k^{-1} \prod_{j=1}^{g}\left(\lambda^{-2}-\bar{c}_{j}\right) \\
k \prod_{j=1}^{g}\left(\lambda^{-2}-c_{j}^{-1}\right) & 0
\end{array}\right) d w
$$

where $k=\prod_{1}^{g} \sqrt{\left|c_{j}\right|}$. For example, the spectral curve for the Wente torus has genus 2 and this potential will compute that surface once the $c_{j}$ are known.

\section{C. Implementation of the DPW Procedure in Software}

Of the three steps in the DPW process, the second requires the most attention. The integration step 1 is performed using a standard fourth order RungeKutta method. We always work with potentials $\xi_{\lambda}$ which are Laurent polynomials in $\lambda$, hence we are always dealing with the Iwasawa decomposition of Laurent polynomial loops. In software, an element of $\Lambda^{\sigma} \mathrm{SL}(2, \mathbb{C})$ is represented as a finite vector, consisting of the coefficients of $\lambda^{-K}$ to $\lambda^{K}$ for some appropriate value of $K$ (typically between 20 and 100). To explicitly construct the Iwasawa factors of $\Phi_{\lambda}$ one proceeds as follows (see [Pressley and Segal 1986]). Let $\mathcal{H}$ denote the Hilbert space $L^{2}\left(S^{1}, \mathbb{C}^{2}\right)$ and let $\mathcal{H}_{+} \subset \mathcal{H}$ be the subspace of maps whose Fourier series possess only non-negative powers of $\lambda$. Define $W=\Phi \mathcal{H}_{+} \subset \mathcal{H}$. Notice that this is the span of $\varphi_{1}$, $\varphi_{2}, \lambda \varphi_{1}, \lambda \varphi_{2}, \ldots$, where $\varphi_{1}, \varphi_{2}$ are the columns of $\Phi$, and that $\lambda W \subset W$ has codimension two. Now compute the orthogonal projections

$$
v_{j}=\operatorname{proj}\left(\varphi_{j}, \lambda W\right) \quad \text { for } j=1,2,
$$

and define $\hat{\varphi}_{j}=\varphi_{j}-v_{j}$ : these two span the space $V=W \cap(\lambda W)^{\perp}$. Finally, let $F_{1}, F_{2}$ be the GramSchmidt orthonormalization of the pair $\hat{\varphi}_{1}, \hat{\varphi}_{2}$, then $F=\left(F_{1}, F_{2}\right)$. It is worth recalling from [Pressley and Segal 1986, p. 126] that on $V$ the $L^{2}$-inner product and the $\mathbb{C}^{2}$-inner product coincide, hence $F$ is unitary on $S^{1}$.

The most time-expensive part of the software version of the DPW procedure arises from computing the projections (2-6). Although they can be found directly (say by Gram-Schmidt orthonormalization of the basis $\left.\varphi_{1}, \varphi_{2}, \lambda \varphi_{1}, \lambda \varphi_{2}, \ldots\right)$, they are computed more efficiently and stably with the following linear method. If $V$ is a finite-dimensional inner product space, $U$ a subspace with basis $u_{1}, \ldots, u_{n}$, and $v \in V$, then $\operatorname{proj}(v, U)=\sum x_{j} u_{j}$ where $x_{1}, \ldots$, $x_{n}$ solve the linear system

$$
\left\langle v-\sum x_{i} u_{i}, u_{j}\right\rangle=0, \quad \text { for } j=1, \ldots, n .
$$

Since this system is Hermitian it can be solved by Cholesky decomposition. Notice that if $\mathcal{H}_{K} \subset \mathcal{H}$ denotes the Laurent polynomials with zero coefficient of $\lambda^{k}$ for $k>K$ then $\lambda^{j} \mathcal{H}_{\mathcal{K}} \perp \mathcal{H}_{K}$ for $j>2 K$, therefore all our calculations take place on finite dimensional subspaces of $\mathcal{H}$.

A further speedup is achieved when the twisted structure of the loop group is exploited. Two elements of $\mathcal{H}$ with opposite polarity are $L^{2}$-orthogonal. In this case, the linear system (2-7) decouples into two simpler Hermitian systems. This also means that the columns of $\widehat{\Phi}=\left(\widehat{\varphi}_{1}, \widehat{\varphi}_{2}\right)$ are already orthogonal, that is, $\widehat{\Phi}$ is unitary. Hence the map $f_{\lambda}$ can be obtained by using $\widehat{\Phi}$ in place of $F$ in the SymBobenko formula and taking the trace-free part of the result.

\section{CMC CYLINDERS}

In this section we will present some new classes of CMC cylinders $f_{\lambda}: \mathbb{C}^{*} \rightarrow \mathbb{R}^{3}$ for which $\xi_{\lambda} \in$ $\Omega_{\mathbb{C}^{*}}^{1,0} \otimes \Lambda_{-1, \infty}^{\sigma} \operatorname{sl}(2, \mathbb{C})$. First let us describe some conditions under which the map $f_{\lambda}$ will be periodic on $\mathbb{C}$ (see also [Dorfmeister and Haak $\geq 2000$ ] for similar results). For any holomorphic potential $\xi_{\lambda} \in \Omega_{\mathbb{C}^{*}}^{1,0} \otimes \Lambda_{-1, \infty}^{\sigma} \operatorname{sl}(2, \mathbb{C})$ the extended holomorphic frame $\Phi_{\lambda}$ has monodromy

$$
\mathcal{M}_{\Phi}(\lambda)=\Phi_{\lambda}(2 \pi) \Phi_{\lambda}^{-1}(0)
$$


where we recall we have chosen to identify $\mathbb{C}^{*}$ with $\mathbb{C} /(2 \pi \mathbb{Z})$. We would like to define a similar notion for the unitary extended frame $F_{\lambda}$ but a priori we do not know that the quantity

$$
\mathcal{M}_{F}(w, \lambda)=F_{\lambda}(w+2 \pi) F_{\lambda}^{-1}(w)
$$

is independent of $w$. However, we can prove the following crucial lemma.

Lemma 3.1. Suppose $\mathcal{M}_{\Phi} \in \Lambda^{\sigma} \mathrm{SU}(2)$. Then $\mathcal{M}_{F}$ is independent of $w \in \mathbb{C}$ and equals $\mathcal{M}_{\Phi}$.

Proof. Since $\Phi_{\lambda}(w+2 \pi)=F_{\lambda}(w+2 \pi) b_{\lambda}(w+2 \pi)$ we have $\mathcal{M}_{\Phi} \Phi_{\lambda}(w)=\mathcal{M}_{F} F_{\lambda}(w) b_{\lambda}(w+2 \pi)$ which implies $\mathcal{M}_{\Phi} F_{\lambda}(w) b_{\lambda}(w)=\mathcal{M}_{F} F_{\lambda}(w) b_{\lambda}(w+2 \pi)$. The result now follows by uniqueness of the Iwasawa decomposition.

Therefore, under the conditions of the lemma, we can sensibly call $\mathcal{M}_{F}$ the monodromy of $F_{\lambda}$ (and in fact this implies $F_{\lambda}^{-1} d F_{\lambda}$ is periodic). Notice that if one knows that the surface is a cylinder then $F_{\lambda}$ necessarily has well-defined monodromy. These observations allow us to formulate an elementary characterization of the conditions under which a periodic potential produces a periodic immersion.

Proposition 3.2. Let $\xi_{\lambda} \in \Omega_{\mathbb{C}^{*}}^{1,0} \otimes \Lambda_{-1, \infty}^{\sigma} \mathrm{sl}(2, \mathbb{C})$ and $\Phi_{\lambda}$ be a solution of $(2-1)$. Suppose $\mathcal{M}_{\Phi} \in \Lambda^{\sigma} \mathrm{SU}(2)$, then, for a given $\lambda_{0} \in S^{1}$, the monodromy of $F_{\lambda}$ satisfies

$$
\mathcal{M}_{F}\left(\lambda_{0}\right)= \pm I
$$

and

$$
\left.\frac{d}{d \lambda} \mathcal{M}_{F}(\lambda)\right|_{\lambda=\lambda_{0}}=0
$$

if and only if the associated family member $f_{\lambda_{0}}$ : $\mathbb{C}^{*} \rightarrow \mathbb{R}^{3}$ obtained by the DPW construction is a CMC immersion of a cylinder.

We will usually work with $\lambda_{0}=1$. Let us now consider some classes of potentials which satisfy the conditions of this proposition.

\section{A. Cylinders with One End Asymptotic to a Delaunay Surface}

An interesting class of surfaces is obtained by perturbing the Delaunay potential $(2-3)$ by a potential on $\mathbb{C}^{*}$ which extends holomorphically into $z=$ 0 . The key to this construction is that $\Phi_{\lambda}^{\text {Del has }}$ monodromy $\mathcal{M}_{\Phi}^{\text {Del }}=\exp \left(2 \pi i A_{\lambda}\right)$, which belongs to $\Lambda^{\sigma} \mathrm{SU}(2)$.
Proposition 3.3. Let

$$
\eta=\sum_{k=0}^{\infty} \eta_{k} z^{k} d z \in \Omega_{\mathbb{C}^{*}}^{1,0} \otimes \Lambda_{-1, \infty}^{\sigma} \operatorname{sl}(2, \mathbb{C})
$$

extend holomorphically to $z=0$ with $\left[\xi^{\mathrm{Del}}, \eta_{0}\right]=0$. Then using the potential $\xi=\xi^{\mathrm{Del}}+\eta$ in $(2-1)$, with an appropriate initial condition, produces a cylinder with one end asymptotic to the Delaunay surface with potential $\xi^{\mathrm{Del}}$.

Proof. Consider the system (2-1) as a first order system of ODE with a regular singular point at $z=0$. We will show below that a solution $\Phi_{\lambda}$ can be written in the form

$$
\Phi_{\lambda}(w)=\Phi_{\lambda}^{\mathrm{Del}}(w) P_{\lambda}\left(e^{i w}\right),
$$

where $P_{\lambda}$ extends holomorphically to $z=0$, with $P_{\lambda}(0)=I$. Given this, we have $\mathcal{M}_{\Phi}=\mathcal{M}_{\Phi}^{\text {Del }}$ since $P_{\lambda}$ has trivial monodromy about $z=0$, so $\mathcal{M}_{\Phi} \in$ $\Lambda^{\sigma} \mathrm{SU}(2)$. By lemma 3.1 we have both $\mathcal{M}_{\Phi}^{\text {Del }}=\mathcal{M}_{F}^{\text {Del }}$ and $\mathcal{M}_{\Phi}=\mathcal{M}_{F}$ hence $\mathcal{M}_{F}=\mathcal{M}_{F}^{\text {Del }}$. Since the Delaunay surface satisfies proposition 3.2 for $\lambda_{0}=1$, so does the perturbed surface.

Now let us verify $(3-1)$. For $P_{\lambda}$ to exist, there must be a solution to the differential equation

$$
d P_{\lambda}=P_{\lambda} \eta_{\lambda}+\left[P, \xi_{\lambda}^{\mathrm{Del}}\right], \quad P_{\lambda}(0)=I .
$$

When we examine the expansion

$$
P_{\lambda}(z)=I+\sum_{k=1}^{\infty} P_{k} z^{k}
$$

we discover we must have

$$
\left(k I+\operatorname{ad} A_{\lambda}\right) P_{k}=\sum_{r+s=k-1} P_{r} \eta_{s} .
$$

Therefore the coefficients $P_{k}$ can be recursively determined provided the operator $k I+\operatorname{ad} A_{\lambda}$ is invertible. The only difficulty occurs for $k=1$, since the non-zero eigenvalues of ad $A_{\lambda}$ are $\pm \nu$ for $\nu=$ $2 \sqrt{ }\left(\frac{1}{4}+\left(\lambda^{-1}-\lambda\right)^{2} a b\right.$ ) (and the reader can easily verify that $|\nu| \leq \sqrt{3}$ for $|\lambda|=1)$. But for $k=1$ we have

$$
\left(I+\operatorname{ad} A_{\lambda}\right) P_{1}=\eta_{0},
$$

which is solved by $P_{1}=\eta_{0}$ since $\left[A_{\lambda}, \eta_{0}\right]=0$. Therefore a solution $P_{\lambda}$ exists of the type required. 
In this class of examples we have more or less complete freedom to specify the location of the umbilics.

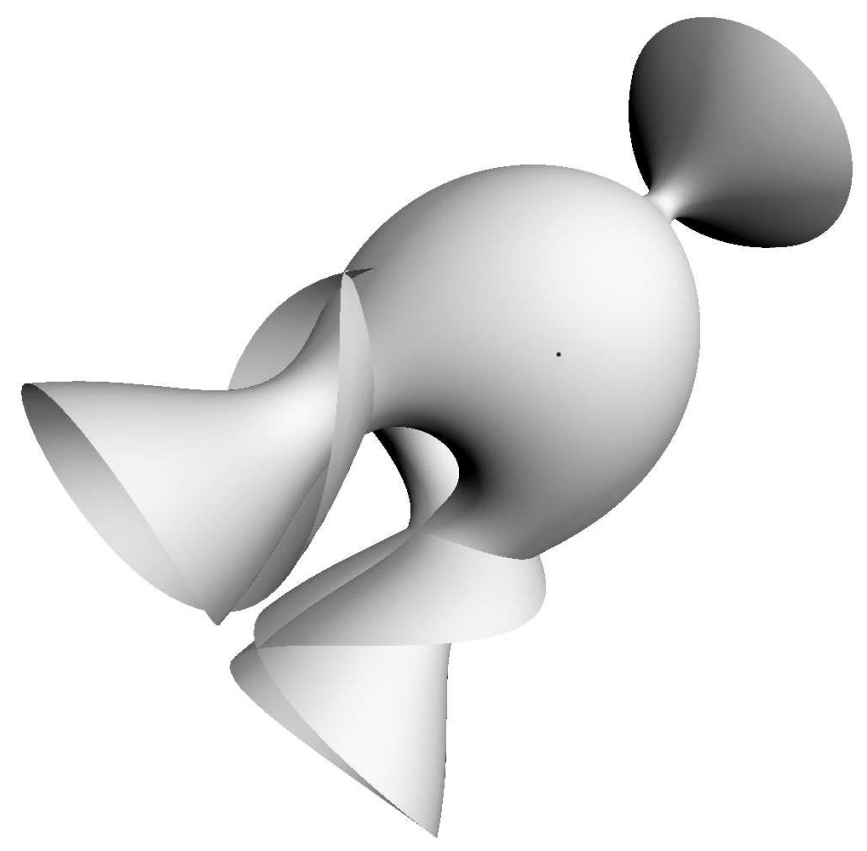

FIGURE 2. CMC cylinder with two umbilics, one marked with a dot. Asymptotically, one end is a Delaunay nodoid with a thin neck and the other is a two-legged Smyth surface. Figure 3 shows a larger piece of this surface.

Example 3. First consider the simplest class of perturbations which produce unbranched surfaces with umbilics. Here we take

$$
\xi_{\lambda}=\left(\begin{array}{cc}
c & a \lambda^{-1}+b \lambda \\
(b-q(z)) \lambda^{-1}+a \lambda & -c
\end{array}\right) \frac{d z}{z},
$$

where $q(z)=k z^{m}$ for $m \geq 2$ and $k$ some constant. By the previous proof, to obtain a cylinder we must use the initial condition $\Phi_{\lambda}(w=0)=P_{\lambda}(z=1)$ in $(2-1)$ : this means first computing the solution $P_{\lambda}(z)$ to $(3-2)$. It is quite remarkable to see that the surfaces obtained appear to be the result of attaching a Delaunay end to the head of a Smyth surface. We have observed that the end opposite to the Delaunay end has $m$ legs which have all the visible characteristics of the Smyth surface legs described above, and possesses an $m$-fold symmetry. It appears that there are $2 m$ planar coordinate lines, one for each angle $n \pi / 2 m$ and the legs develop around those for $n$ even. The umbilics, which lie at the $m$-th roots of $b / k$, lie on these lines just before the first selfintersections (as we move away from the Delaunay end). Indeed, each Smyth surface lies in a one real parameter family of surfaces with potential $(3-3)$. To see this, observe that the gauge transformation (2-5) transforms the Smyth surface potential as

$$
\lambda^{-1}\left(\begin{array}{cc}
0 & 1 \\
p(z) & 0
\end{array}\right) d z \mapsto\left(\begin{array}{cc}
0 & \lambda^{-1} \\
z^{2} p(z) \lambda^{-1}+\lambda & 0
\end{array}\right) \frac{d z}{z},
$$

which is $(3-3)$ with $a=1, b, c=0$ and $q(z)=$ $-z^{2} p(z)$. Therefore it makes sense to think of the surfaces we see as deformations of the Smyth surfaces, where the intrinsic $S^{1}$-symmetry has been broken by the bifurcation of the multiple umbilic at $z=0$ into $m$ umbilics at equal distance from the origin and at equal angles. Because of this, we conjecture that these cylinders are complete and proper immersions for which the end for $z \rightarrow \infty$ is bounded by a cone.

The $m$-fold rotational symmetry is explained by reference to the earlier discussion at the end of Section $2 \mathrm{~A}$. Let $\nu \in \operatorname{Aut}(\mathbb{C})$ denote translation by $2 \pi / m$. This induces on $\mathbb{C}^{*}$ a rotation through this angle. Then $\nu^{*} \eta=\eta$, so the same is true for $\xi_{\lambda}$. A careful examination of the series expansion of $(3-2)$ shows that this implies $\nu^{*} P_{\lambda}=P_{\lambda}$. It follows that

$$
\Phi_{\lambda}\left(\nu\left(w_{0}\right)\right) \Phi_{\lambda}\left(w_{0}\right)^{-1} \in \Lambda^{\sigma} \mathrm{SU}(2)
$$

for $w_{0}=0$; therefore we have all the conditions for this symmetry to exhibit itself on the surface.

More general types of perturbations than (3-3) do not seem to alter the end behavior a great deal. Certainly taking $q(z)$ to be any polynomial has the effect one expects from knowledge of the generalized Smyth surfaces: the number of $\operatorname{legs}$ is $\operatorname{deg}(q)$ and their direction depends in some way upon the roots of $q(z)-b$. If we consider perturbations at higher powers of $\lambda$ we can obtain surfaces with no umbilics but they still appear to have the same end behavior.

Example 4. The form of the potential (3-3) made us think that to some extent we may be able to treat holomorphic potentials like building blocks to patch two types of end behavior together. Therefore we considered potentials of the form $\xi_{\lambda}-\nu^{*} \hat{\xi}_{\lambda}$ where $\nu(z)=1 / z$ and $\xi_{\lambda}, \hat{\xi}_{\lambda}$ have the same Delaunay end. The rationale here is that this might attach the surfaces for $\xi_{\lambda}$ and $\nu^{*} \hat{\xi}_{\lambda}$ together along a Delaunay tube centered at the image of $|z|=1$ (we can always make this lie on the Delaunay end by suitable scaling). For 

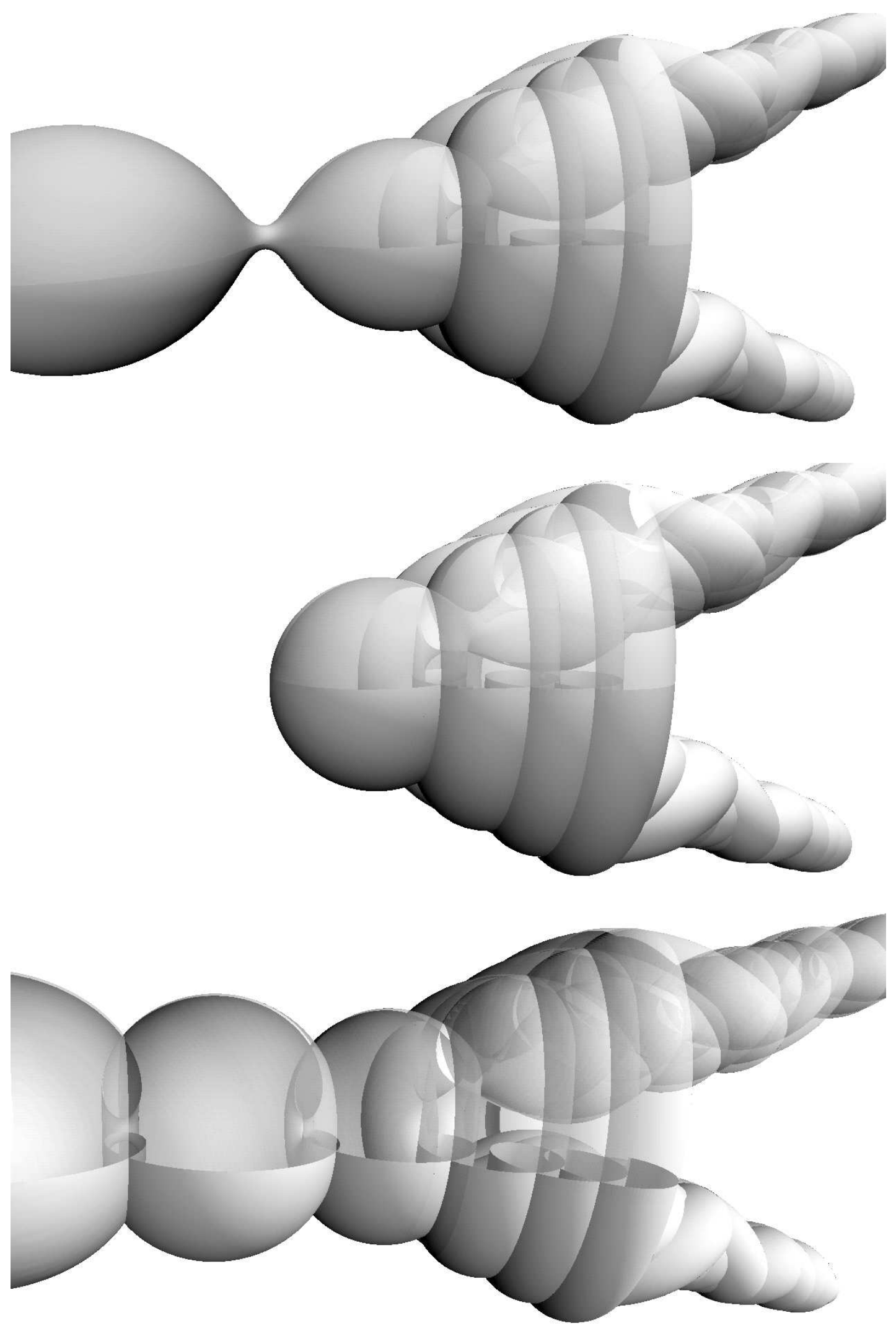

FIGURE 3. Two-legged Smyth surfaces with Delaunay heads. The Smyth surface (middle) has a sphere-like head and is a singular boundary between the unduloidal and nodoidal examples. 


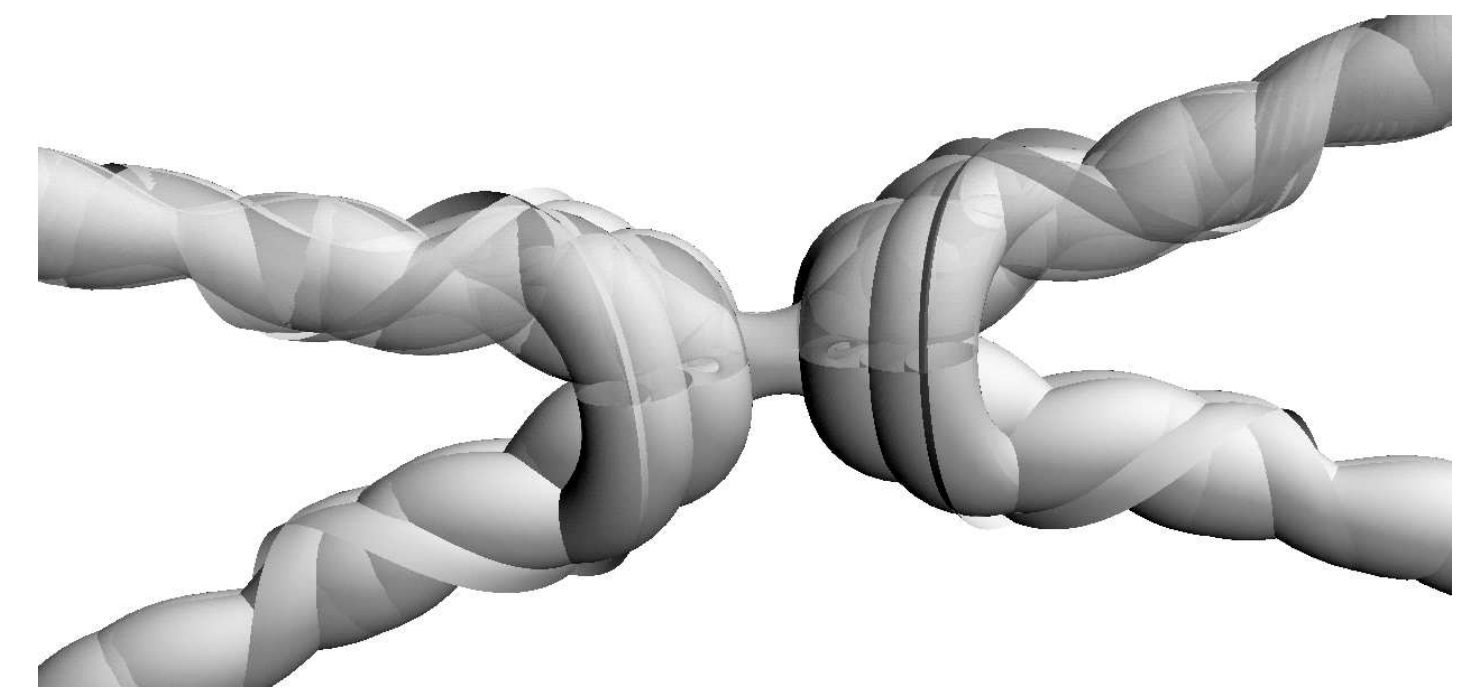

FIGURE 4. The double Mr. Bubble is two two-legged Smyth surfaces joined by an unduloidal neck.

$\xi_{\lambda}, \hat{\xi}_{\lambda}$ of the type $(3-3)$ this amounts to examining potentials of the form $(3-3)$ where now

$$
q(z)=z^{2} r(z)+z^{-2} s(1 / z)
$$

and $r(z), s(z)$ are entire functions. Although we do not claim that the resultant surfaces close into cylinders, the experiments show that they are very close to closing and are quite stable to perturbations of the coefficients of $r$ and $s$. As one would hope, each end has the expected number of legs: $\operatorname{deg}(r)$ for the end near $z=0$ and $\operatorname{deg}(s)$ for the end near infinity. We believe that cylinders of this type exist with the correct choice of initial condition for $(2-1)$.

\section{B. CMC Cylinders with a Closed Planar Geodesic}

Proposition 3.2 gives conditions on the monodromy of the extended unitary frame $F_{\lambda}$ which are in general hard to verify, since both integration of $(2-1)$ and the subsequent Iwasawa decomposition cannot usually be performed explicitly. Here we will work with a class of potentials for which $(2-1)$ can be integrated explicitly at least over the unit circle. We will choose $\xi_{\lambda}$ to be $\Lambda^{\sigma} \mathrm{su}(2)$-valued on the unit circle $|z|=1$ in $\mathbb{C}^{*}$. It follows that the solution $\Phi_{\lambda}$ to $(2-1)$ (with $g=I, w_{0}=0$ ) will take values in $\Lambda^{\sigma} \mathrm{SU}(2)$, whence $\Phi_{\lambda}=F_{\lambda}$ along $\mathbb{R} \subset \mathbb{C}$ and by Lemma 3.1 the monodromy $\mathcal{M}_{F}$ is well-defined. It is not hard to see that $\xi$ is $\Lambda^{\sigma} \mathrm{su}(2)$-valued on the unit circle if and only if $\rho^{*} \xi=-\overline{\xi^{t}}$ where $\rho(z)=1 / \bar{z}$. Since it is always possible to gauge away the diagonal terms of a potential which is $\Lambda^{\sigma} \mathrm{su}(2)$-valued on
$S^{1}$, we may assume without loss of generality that $\xi_{\lambda}$ is of the form

$$
\xi_{\lambda}=\left(\begin{array}{cc}
0 & \alpha \lambda^{-1}+\beta \lambda \\
-\overline{\rho^{*} \beta} \lambda^{-1}-\overline{\rho^{*} \alpha} \lambda & 0
\end{array}\right)
$$

with $\alpha, \beta \in \Omega_{\mathbb{C}^{*}}^{1,0}$.

In the first class of potentials of this type we will also insist that $\alpha, \beta$ both satisfy $\overline{\rho^{*} \alpha}=-\alpha$. In this case, under the conditions of the next proposition, the image of the unit circle is a planar geodesic which contains the umbilics: we exhibit some examples in Figure 7. For the purposes of the next proposition, let $z(t)$ denote the contour $t \mapsto e^{i t}$ in $\mathbb{C}^{*}$.

Proposition 3.4. Let $\alpha, \beta \in \Omega_{\mathbb{C}^{*}}^{1,0}$ satisfy

1. $\overline{\rho^{*} \alpha}=-\alpha, \overline{\rho^{*} \beta}=-\beta$,

2. $\int_{S^{1}}(\alpha+\beta) \in \pi i \mathbb{Z}$ and

3. $\int_{S^{1}}(\alpha-\beta) \exp \left(2 \int_{1}^{z(t)}(\alpha+\beta)\right)=0$.

Then

$$
\xi_{\lambda}=\left(\begin{array}{cc}
0 & \alpha \lambda^{-1}+\beta \lambda \\
\beta \lambda^{-1}+\alpha \lambda & 0
\end{array}\right)
$$

is the potential for a CMC cylinder with umbilics at the zeroes of $\beta$. Further, the plane containing the image of the unit circle is a plane of reflective symmetry.

Proof. For $\lambda=1$, the solution of

$$
d \Phi_{1}=\Phi_{1} \xi_{1}, \quad \Phi_{1}(0)=I
$$


along $t \in \mathbb{R} \subset \mathbb{C}$ is given by $\Phi_{1}(t)=\exp \int_{1}^{z(t)} \xi_{1}$. Therefore

$$
\mathcal{M}_{\Phi}(1)=\exp \left(\int_{S^{1}}(\alpha+\beta)\left(\begin{array}{ll}
0 & 1 \\
1 & 0
\end{array}\right)\right) .
$$

Since $\Phi_{\lambda}=F_{\lambda}$ along $\mathbb{R}$ the first monodromy condition from proposition 3.2 becomes $\mathcal{M}_{\Phi}= \pm I$, which is equivalent to

$$
\int_{S^{1}}(\alpha+\beta)=k \pi i, \quad \text { where } k \in \mathbb{Z} .
$$

Similarly, it is straightforward to check that the second monodromy condition of proposition 3.2 is implied by

$$
\left.\int_{S^{1}} \Phi_{\lambda} \frac{\partial \xi}{\partial \lambda} \Phi_{\lambda}^{-1}\right|_{\lambda=1}=0
$$

A computation yields

$$
\left.\int_{S^{1}} \Phi_{\lambda} \frac{\partial \xi}{\partial \lambda} \Phi_{\lambda}^{-1}\right|_{\lambda=1}=\int_{S^{1}}(\alpha-\beta)\left(\begin{array}{ll}
\sinh \omega & -\cosh \omega \\
\cosh \omega & -\sinh \omega
\end{array}\right),
$$

where $\omega=2 \int_{1}^{z(t)}(\alpha+\beta)$. Using the reality conditions on $\alpha, \beta$, this integral vanishes precisely when $\int_{S^{1}}(\alpha-\beta) \exp \omega=0$.

Example 5. The simplest example is obtained with the forms

$$
\alpha=-\frac{d z}{2 z}, \quad \beta=\left(\frac{1}{2}+\kappa\left(z^{-1}+z\right)\right) \frac{d z}{z} .
$$

Here the constant $\kappa$ must satisfy $J_{0}(4 \kappa)=0$ where $J_{0}$ is the Bessel function of order zero. To see this observe that if we parametrize the unit circle by $z(t)=e^{i t}$ we have

$$
\begin{aligned}
\int_{S^{1}}(\alpha-\beta) \exp & \left(2 \int_{1}^{z}(\alpha+\beta)\right) \\
= & -\int_{0}^{2 \pi}(1+2 \kappa \cos t) \exp (4 i \kappa \sin t) d t \\
& =-2 J_{0}(4 \kappa) .
\end{aligned}
$$

It follows that we have a discrete family of immersed CMC cylinders indexed by the zeroes of $J_{0}(4 \kappa)$. Further, $\overline{\xi(1 / \bar{z})}=-\xi(z)^{t}$ and $\overline{\xi(\bar{z})}=\xi(z)$. Therefore each cylinder in this family has two planar symmetries: one plane containing the image of the unit circle and the other containing the image of the real axis. From the graphics we observe that, near the planar geodesic, the image of the the positive real axis resembles a profile curve of a nodoid while the image of the negative real axis resembles the profile
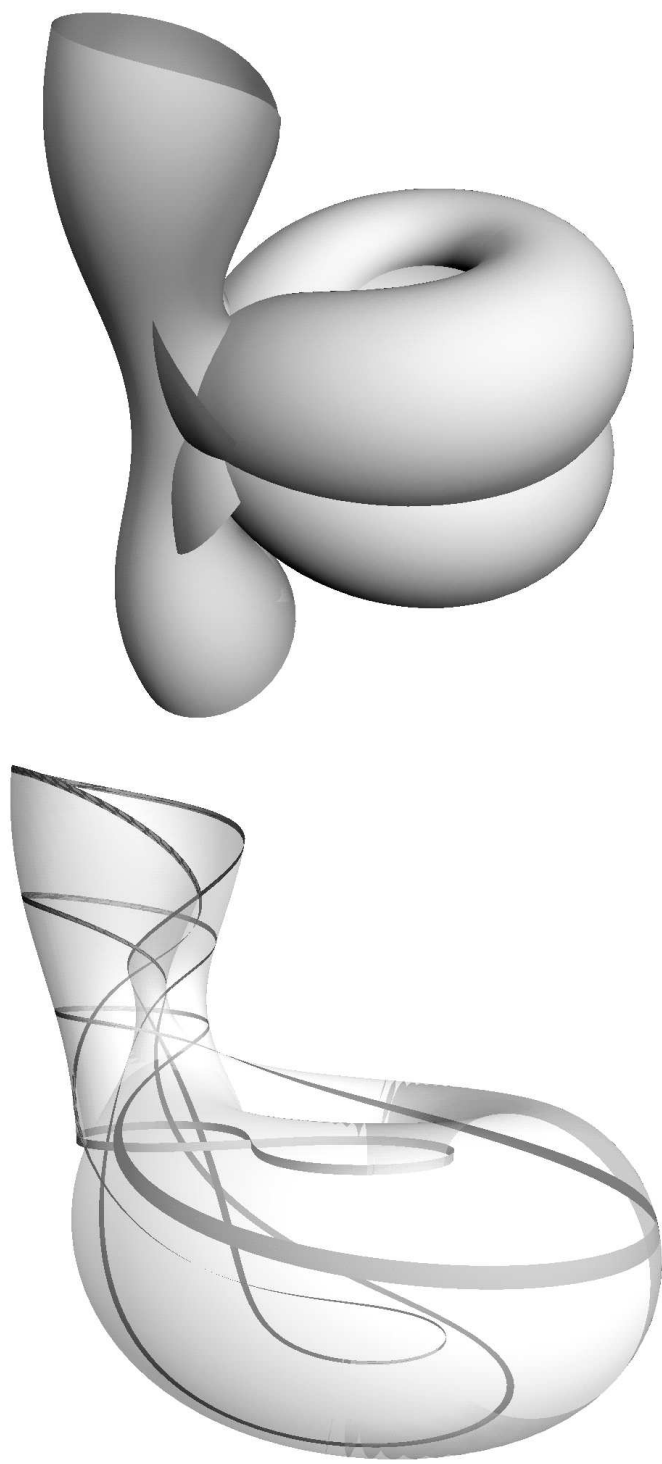

FIGURE 5. This CMC cylinder has the appearance of an unduloid conjoined with a nodoid. The figure-eight in the transparent image is the planar geodesic across which the surface has reflective symmetry. As it evolves toward an end, one of its loops sweeps out half of the unduloid, the other traces the opposite half-nodoid.

curve of an unduloid. Figure 5 displays aspects of the surface for the first positive root of $J_{0}(4 \kappa)$.

More examples can be obtained using the following method. The first monodromy condition is simply $\operatorname{res}_{0}(\alpha+\beta) \in \frac{1}{2} \mathbb{Z}$, where we consider $\alpha, \beta$ as 1 -forms on $\mathbb{C}$ with an isolated singularity at $z=0$. For the second condition, set $\omega=(\alpha-\beta) \exp \left(2 \int \alpha+\beta\right)$, then $\omega$ is a 1 -form on $\mathbb{C}^{*}$ by the first monodromy condition. It is straightforward to show that, for any 
1-form $\omega$, the residue $\operatorname{res}_{0} \omega=0$ vanishes whenever $\nu_{a}^{*} \omega=a^{k} \omega$ for $\nu_{a}(z)=a z$ with $a^{k} \neq 1$. In particular we consider the case where $a$ is a primitive $n$-th root of unity. Then $\nu_{a}^{*} \omega=a^{k} \omega$, and the reality conditions are satisfied, if $\alpha, \beta$ are of the form

$$
\sum_{j \in \mathbb{N} \cup\{0\}}\left(c_{j} z^{n j}+\bar{c}_{j} z^{-n j}\right) \frac{d z}{z}, \quad \text { for } c_{j} \in \mathbb{C},
$$

with $2 \operatorname{res}_{0}(\alpha+\beta) \in n \mathbb{Z}+k$ for $\operatorname{gcd}(k, n)=1$. In this case the potential $(3-4)$ possesses the symmetries $\nu_{a}^{*} \xi_{\lambda}=\xi_{\lambda}$ and $\overline{\rho^{*} \xi_{\lambda}}=-\xi_{\lambda}^{t}$. These imply that the surface has an $n$-fold rotational symmetry (since $\Phi_{\lambda}$ is $\Lambda^{\sigma} \mathrm{SU}(2)$-valued over $\left.|z|=1\right)$. Further, if $\alpha, \beta$ also satisfy $\alpha(\bar{z})=\alpha(z)$ then the surfaces will have $n$ extra planes of reflective symmetry (for example, see Figure 6).

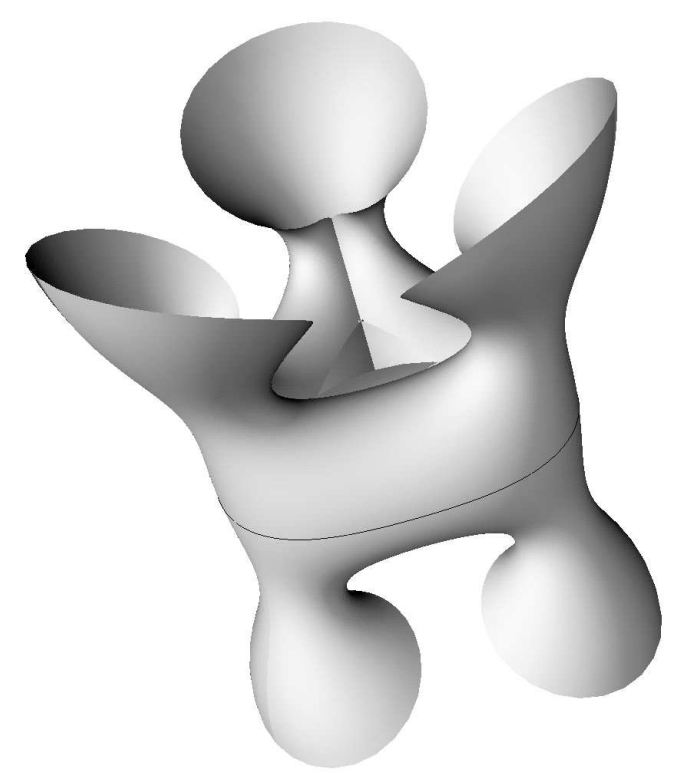

FIGURE 6. The planar geodesic of this CMC cylinder, marked in black, lies near the beginning of the sequence shown in Figure 7.

Figure 7 shows a sequence of planar geodesic crosssections for CMC cylinders with potential (3-4) for $\alpha=\frac{1}{2} d z / z$ and $\beta=c\left(z^{3}+z^{-3}\right) d z / z$, where $c \in \mathbb{R}$. For $c=0$ we obtain the round sphere. As $c$ increases (left to right) the curves acquire more loops.

\section{C. Other CMC Cylinders}

Another class of examples is obtained by asking that the holomorphic potential satisfy $\xi_{1}=0$ and $\overline{\rho^{*} \xi_{\lambda}}=$ $-\xi_{\lambda}^{t}$ for $\rho(z)=1 / \bar{z}$.

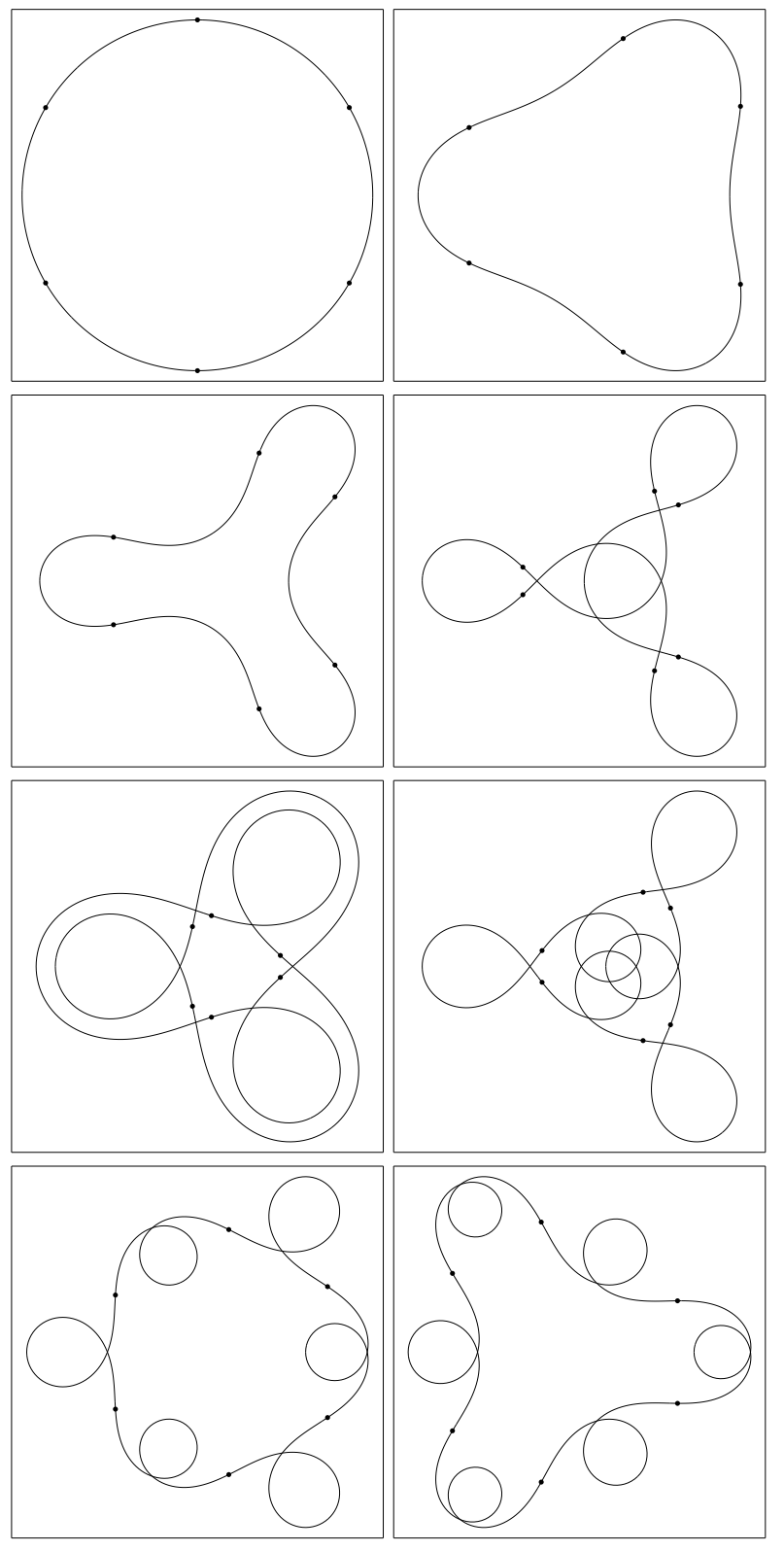

FIGURE 7. Planar geodesics which are the cross sections of a CMC cylinder family. The dots mark the six umbilic points.

Proposition 3.5. Let $\alpha \in \Omega_{\mathbb{C}^{*}}^{1,0}$ with $\int_{S^{1}} \alpha=0$. Then

$$
\xi_{\lambda}=\left(\begin{array}{cc}
0 & \alpha \\
\rho^{*} \alpha & 0
\end{array}\right)\left(\lambda^{-1}-\lambda\right)
$$

is the potential for a CMC cylinder with umbilics at the zeroes of $\overline{\rho^{*} \alpha}$ and branch points at the zeroes of $\alpha$.

Proof. Since $\xi_{1}(z)=0$, the solution $\Phi_{\lambda}$ to $(2-1)$ with $\Phi_{\lambda}(0)=I$ has $\Phi_{1}=I$. As above, $\Phi_{\lambda}=F_{\lambda}$ over the unit circle and we deduce $\mathcal{M}_{F}=I$, so the first 
monodromy condition of proposition 3.2 is satisfied. The second monodromy condition follows from

$$
\left.\int_{S^{1}} \Phi_{\lambda} \frac{\partial \xi}{\partial \lambda} \Phi_{\lambda}^{-1}\right|_{\lambda=1}=-2 \int_{S^{1}}\left(\begin{array}{cc}
0 & \alpha \\
\rho^{*} \alpha & 0
\end{array}\right)=0
$$

as in the proof of proposition 3.4.

The cylinders generated by these potentials have constant frame $F_{\lambda}=I$ over the unit circle. This means that the Gauss map is constant along the image of the unit circle so that this lies on a single tangent plane to the surface.

Example 6. First, this class contains all Delaunay nodoids. They arise if we take any $s \in \mathbb{R} \backslash\{-1\}$ and set $\alpha=z^{s+1} d z$. The explanation for this lies in the gauge transformation

$\left(\begin{array}{cc}-s / 2 & \lambda^{-1}-\lambda \\ -\left(\lambda^{-1}-\lambda\right) & s / 2\end{array}\right) \frac{d z}{z} \mapsto\left(\begin{array}{cc}0 & z^{s} \\ -z^{-s} & 0\end{array}\right)\left(\lambda^{-1}-\lambda\right) \frac{d z}{z}$ achieved by gauging the left-hand potential by

$$
\gamma=\left(\begin{array}{cc}
e^{s w / 2} & 0 \\
0 & e^{-s w / 2}
\end{array}\right)
$$

where $z=e^{w}$. This left-hand potential is $-s \xi_{\lambda}^{\mathrm{Del}}$ with $a=-b=-1 / s$ and $c=1 / 2$

By contrast, if we take $\alpha=(1+z) d z$ we obtain the surface in Figure 8. This example displays the characteristic features of the cylinders in this class.

More generally, if $\alpha=p(z) d z$ for a polynomial $p(z)$, we have observed that the resulting surface has $\operatorname{deg}(p)$ legs emerging within a nodoid-like sheath. Experiments suggest that all surfaces in this class are bounded by the outer nodoid-like surface.

\section{CONCLUDING REMARKS}

It is difficult to convey in static pictures the intuition gained by being able to rotate, cut away and zoom in on these surfaces. One feature which struck us was the ubiquity of nodoidal and unduloidal features in the ends. In fact, the Smyth end itself, which at first looks impossibly complicated, appears to have the following simple description. Consider the 2-legged Smyth end. Divide the region $|z|>1$ into its four quadrants. The lines at angles $0, \pi$ are mapped to unduloidal-like profiles, which decay in amplitude as the radius increases. The line at angles $\pi / 2,3 \pi / 2$ are mapped to nodoidal-like profiles which become more circular as the radius increases.
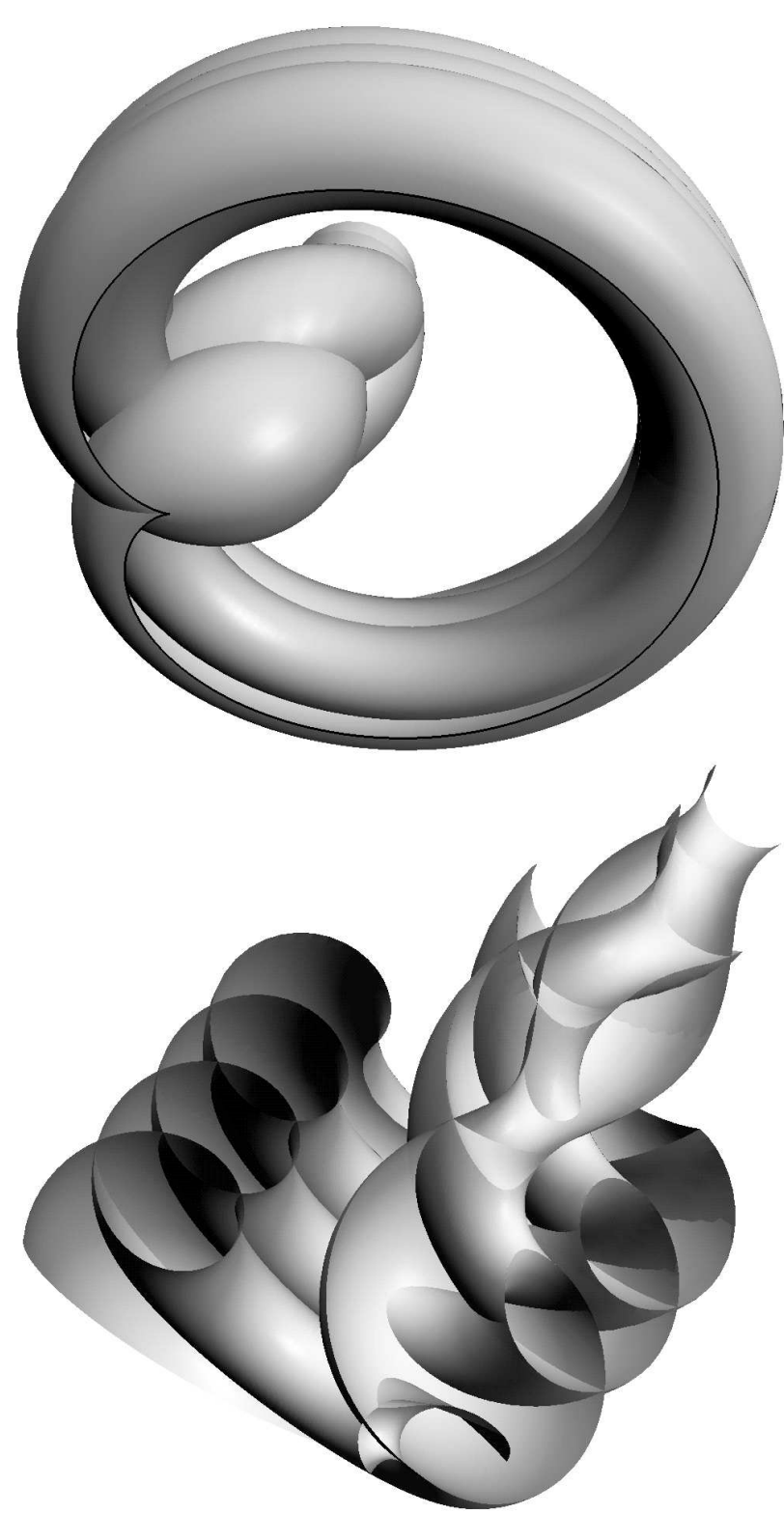

FIGURE 8. This CMC cylinder is tangent to a plane along the black curve in the solid figure.

Between these lines the surface must interpolate between an unduloid and a nodoid. It does so in a way which strongly resembles the way a Delaunay unduloid unravels and wraps up into a Delaunay nodoid as it moves through the associated family.

The surfaces introduced in Sections $3 \mathrm{~B}$ and $3 \mathrm{C}$ have a similar description as we rotate around $\mathbb{C}^{*}$. But their behavior as the radius increases or decreases is quite different. Figures 5 and 8 lead us to ask: are either of these surfaces bounded by a standard cylinder? 
For the surface with a planar geodesic in Figure 5 , as $|z|$ increases from $|z|=1$ (or as it decreases) each circle is stretched in two opposite directions in 3-space. Since the image of circles of constant $|z|$ appear to pass through the central plane of reflection not far from the planar curve, it is not yet settled whether these examples are properly immersed.

On the other hand, the surface in Figure 8 seems to be made by translating the same shape as the radius $|z|$ increases and decreases from $|z|$, although this cannot be literally true since there is only one umbilic (and branch point): it lies at $z=-1$. This suggests that this map is proper.

It seems that these surfaces give two new types of end behavior which, although they are immersed, do not appear to be significantly more complicated than the Smyth surfaces.

\section{APPENDIX BY NICHOLAS SCHMITT: K-NOIDS}

\section{A. Introduction}

A problem of fundamental importance in the theory of constant mean curvature surfaces is the classification of complete CMC surfaces with ends asymptotic to Delaunay surfaces ( $k$-noids). Examples have been constructed using the conjugate cousin construction of minimal surfaces in $S^{3}$ [Große-Brauckmann 1993; Große-Brauckmann et al. 1999]. The almost embedded CMC surfaces with genus zero and three ends (triunduloids) have been classified:

Theorem (Große-Brauckmann, Kusner, Sullivan). Triunduloids are classified by triples of distinct labeled points in the two-sphere (up to rotations); the spherical distances of points in the triple are the necksizes of the unduloids asymptotic to the three ends. The moduli space of triunduloids is therefore homeomorphic to an open three-ball.

Specifying DPW potentials and initial conditions to produce $k$-noids will require a careful analysis of the Riemann-Hilbert problem. In the genus-zero case, trinoids can be constructed by choosing DPW potentials whose monodromy is computable using hypergeometric functions, according to J. Dorfmeister and $\mathrm{H}$. $\mathrm{Wu}$ (private communication). For potentials with more than three poles, the Riemann-Hilbert problem cannot be solved with hypergeometric functions, but one still expects to be able to prove exis- tence for the various Delaunay-type end configurations and describe their moduli.

In this appendix we conjecture, on the basis of computer experiments, the existence of three families of genus-zero $k$-noids. The meromorphic potentials for these examples are constructed as the linear superposition of Delaunay potentials.

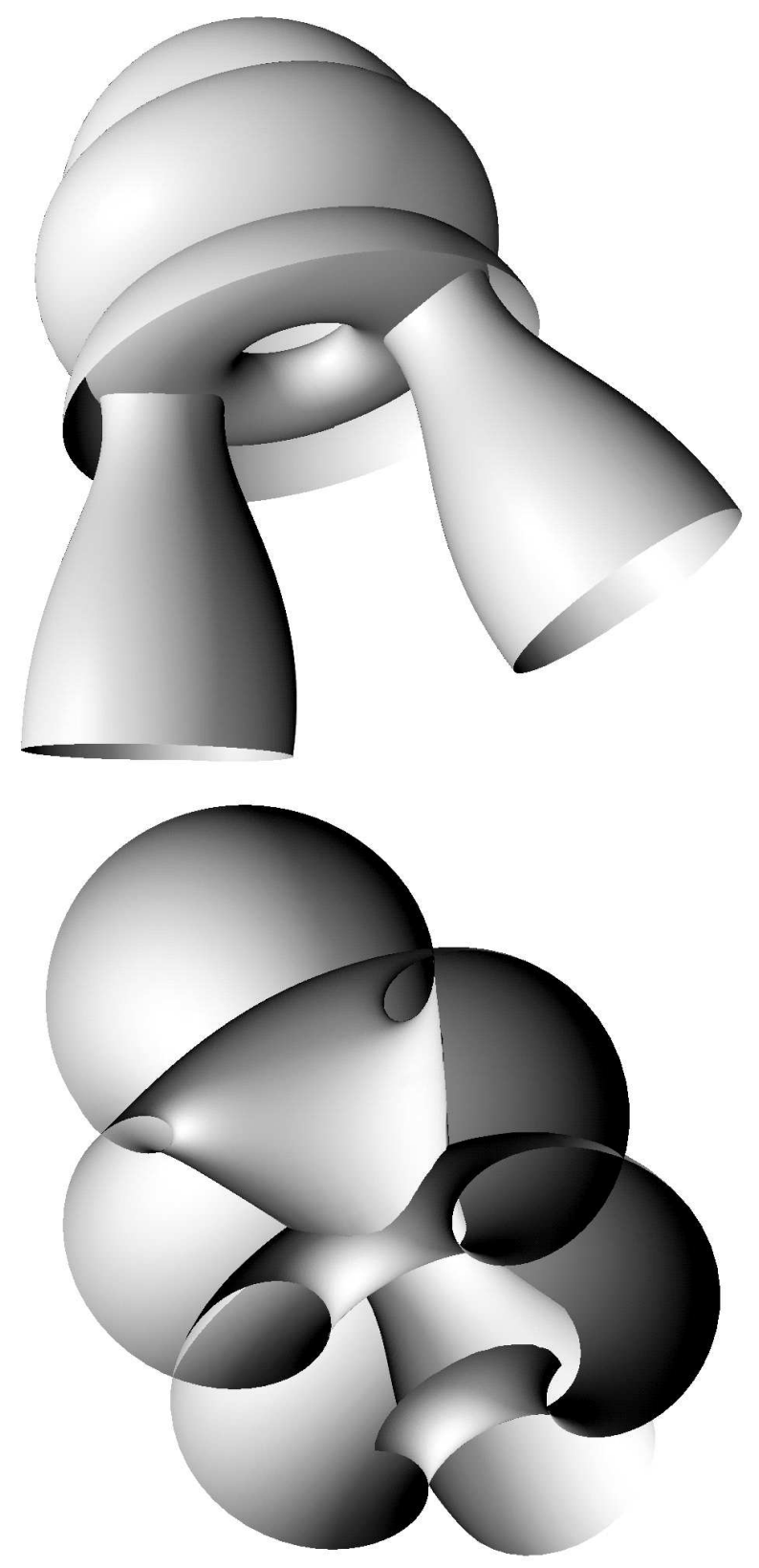

FIGURE 9. Two views of an isosceles 3 -noid with one nodoid end; all three ends extend infinitely downward. In contrast to the triunduloids, the two umbilic points lie not symmetrically about the mirror plane but on it. 


\section{B. Balancing}

As a prerequisite for specifying the meromorphic potentials for the conjectured families, we discuss the balancing formula for $k$-noids [Bobenko 1994; Korevaar et al. 1989; Kusner 1991].

Theorem 5.1 (Balancing Formula). Let $S$ be an k-noid with monodromies

$$
M_{n}=U_{n} \exp \left(2 \pi i A_{n}\right) U_{n}^{-1} \quad(n=1 \ldots k),
$$

with $U_{n} \in \Lambda^{\sigma} \mathrm{SU}(2)$ and

$$
A_{n}=\left(\begin{array}{cc}
c_{n} & a_{n} \lambda^{-1}+\bar{b}_{n} \lambda \\
b_{n} \lambda^{-1}+\bar{a}_{n} \lambda & -c_{n}
\end{array}\right)
$$

and $\mu_{n}=\left(\left|a_{n}+\bar{b}_{n}\right|^{2}+c_{n}^{2}\right)^{1 / 2} \in \frac{1}{2} \mathbb{Z}$ as in (2-3). Then

$$
\left.\sum \frac{a_{n} b_{n}}{\mu_{n}^{2}} U_{n} A_{n} U_{n}^{-1}\right|_{\lambda=1}=0 .
$$

Proof. With $\lambda=e^{i \theta}$, apply $\partial^{2} /\left.\partial \theta^{2}\right|_{\theta=0}$ to $\prod M_{n}=I$.

In interpreting the balancing formula [Bobenko 1991; Kusner 1991], each summand can be thought of as a force vector along a unit-length asymptotic end axial direction, scaled, up to a proportionality constant, by a weight:

$$
\begin{aligned}
\text { axis } & =U(i A / \mu) U^{-1} \in \operatorname{su}(2) \cong \mathbb{R}^{3}, \\
\text { weight } & =2 a b / \mu \in \mathbb{R}, \\
\text { force } & =\text { weight } \cdot \text { axis. }
\end{aligned}
$$

The end weight is equal to $m H^{2} r_{+} r_{-}$, where $m$ is the end's wrapping number and $r_{ \pm}$are the signed neck/bulge radii as in (2-4). The weights of unduloid/nodoid ends are respectively positive/negative.

As a corollary of the balancing formula we have the triangle-type inequalities

$$
\left|w_{n}\right| \leq \sum_{j \neq n}\left|w_{j}\right| \quad(n=1, \ldots, k) .
$$

Applying $\partial^{3} /\left.\partial \theta^{3}\right|_{\theta=0}$ to $\prod M_{n}=I$ leads to a torque balancing formula.

\section{C. Genus-Zero k-noids}

We conjecture the existence of two families of trinoids and a discrete family of $k$-noids.

Conjecture 5.2 (Triunduloids). The triunduloids are $o b$ tained from the meromorphic potential $\xi\left(a_{1}, a_{2}, a_{3}, c\right)$
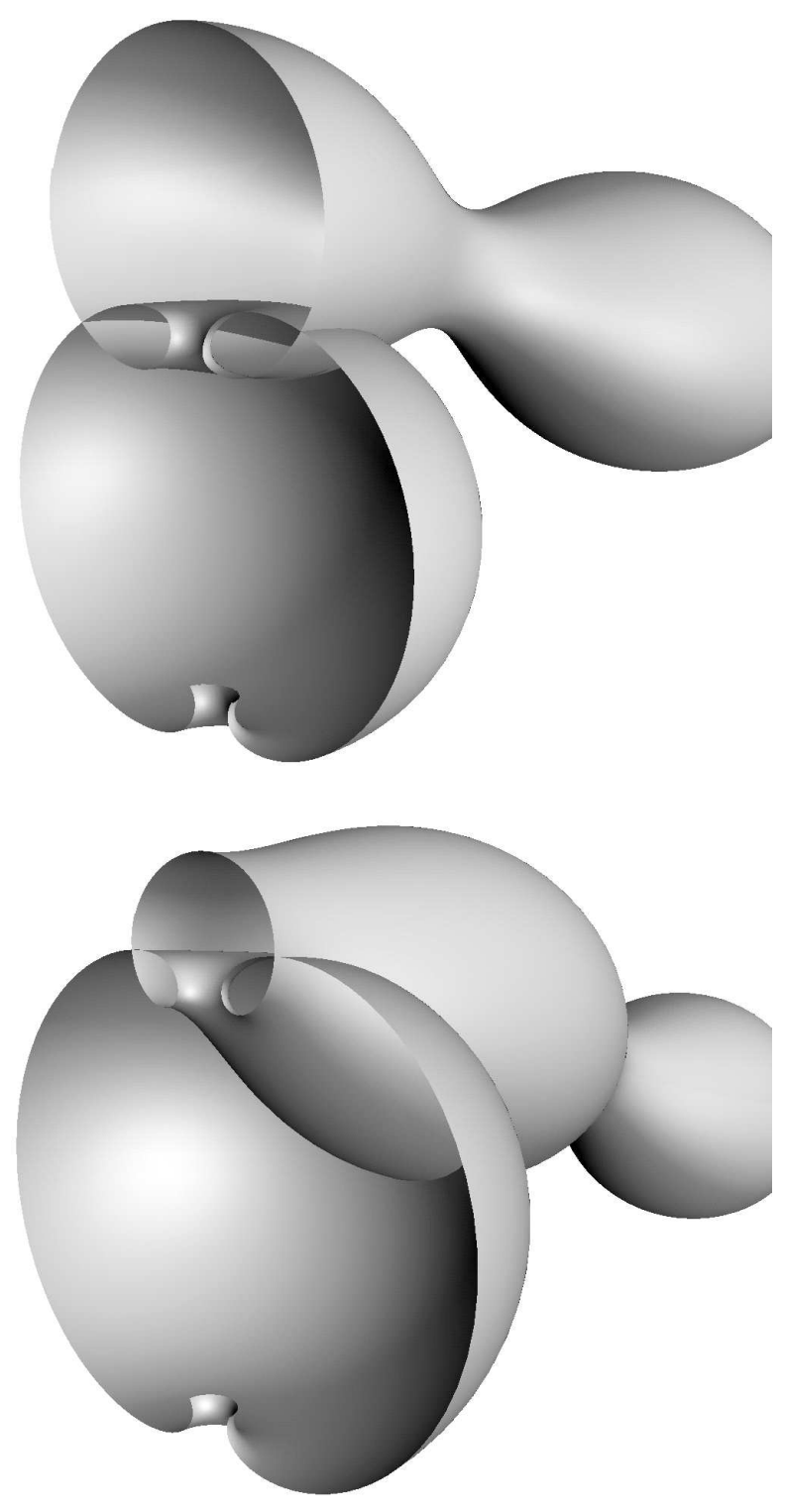

FIGURE 10. Two isosceles 3-noids with one nodoid end, sliced by a mirror plane. The corresponding ends of the two surfaces have the same neck radii but opposite phase: the top example has a bulge at the center, the bottom one a neck.

(see Definition 5.4) subject to weight constraints

$$
w_{1}>0, \quad w_{2}>0, \quad w_{3}>0 .
$$

Conjecture 5.3 (Trinoids with one nodoid end). There exists a family of 3-noids with one nodoid and two unduloid ends. These are obtained from the meromorphic potential $\xi\left(a_{1}, a_{2}, a_{3}, c\right)$ (see Definition 5.4) subject to weight constraints $w_{1}>0, w_{2}>0, w_{3}<0$, $\left|w_{3}\right| \leq\left|w_{1}\right|+\left|w_{2}\right|$. 
Definition 5.4 (Family of trinoid potentials). Let $e_{1}, e_{2}$, $e_{3} \in \mathbb{C P}$ be fixed distinct points. Let $a_{1}, a_{2}, a_{3} \in$ $\mathbb{R} \backslash\{0\}$, and $s, c \in \mathbb{R}$ satisfy

$$
s=a_{1}+a_{2}+a_{3}, \quad s^{2}+c^{2}=\frac{1}{4} .
$$

The meromorphic $\Lambda^{\sigma} \mathrm{sl}(2, \mathbb{C})$-valued differential $\xi=$ $\xi\left(a_{1}, a_{2}, a_{3}, c\right)$ is defined uniquely by these conditions:

(i) $\xi$ has simple poles only at $e_{1}, e_{2}, e_{3}, p \in \mathbb{C P}$.

(ii) With $A_{\lambda}$ denoting the Delaunay residue as in $(2-3)$, the residues of $\xi$ are

$$
\begin{aligned}
\operatorname{res}_{e_{1}} \xi & =A_{\lambda}\left(-s+a_{1},-a_{1},-c\right), \\
\operatorname{res}_{e_{2}} \xi & =A_{\lambda}\left(a_{2}, s-a_{2}, c\right), \\
\operatorname{res}_{e_{3}} \xi & =A_{\lambda}\left(a_{3}, s-a_{3}, c\right), \\
\operatorname{res}_{p} \xi & =A_{\lambda}(0,-s,-c) .
\end{aligned}
$$

(iii) the Hopf differential corresponding to $\xi$ has neither pole nor zero at $p$; with the above residues, this condition specifies $p$ uniquely.

The end weights of the trinoid induced by

$$
\xi\left(a_{1}, a_{2}, a_{3}, c\right)
$$

are $w_{k}=4 a_{k}\left(a_{i}+a_{j}\right)$, with $\{i, j, k\}=\{1,2,3\}$.

Conjecture 5.5 (Maximal equilateral k-noids). For each integer $k \geq 2$ there exists an equilateral $k$-noid with meromorphic potential $\xi_{k}$ defined by

$$
\begin{aligned}
\xi_{k} & =\left(\begin{array}{cc}
0 & \alpha_{k} \lambda^{-1}+\beta_{k} \lambda \\
\beta_{k} \lambda^{-1}+\alpha_{k} \lambda & 0
\end{array}\right), \\
\alpha_{k} & =\frac{\left(z^{k-1}-1\right) d z}{2(z-1)\left(z^{k}-1\right)}, \\
\beta_{k} & =\frac{(k-1) z^{k-2}(z-1) d z}{2\left(z^{k-1}-1\right)\left(z^{k}-1\right)} .
\end{aligned}
$$

Cone Points. The conjectured $k$-noid potentials have, besides the $k$ poles inducing ends, $k-2$ extra poles, which we will call cone points, that are not ends (or umbilics) on the CMC surface. These arise from the fact that the meromorphic differential $\beta$ in the potential

$$
\xi=\left(\begin{array}{cc}
\gamma & \alpha \lambda^{-1}+\beta \lambda \\
\beta \lambda^{-1}+\alpha \lambda & -\gamma
\end{array}\right)
$$

has $k$ simple poles at the ends and a total of $2 k-4$ zeros at umbilics, and must therefore have $k-2$ more poles so that the algebraic sum is -2 . At these cone points, $\alpha$ is constructed to have zeros so that
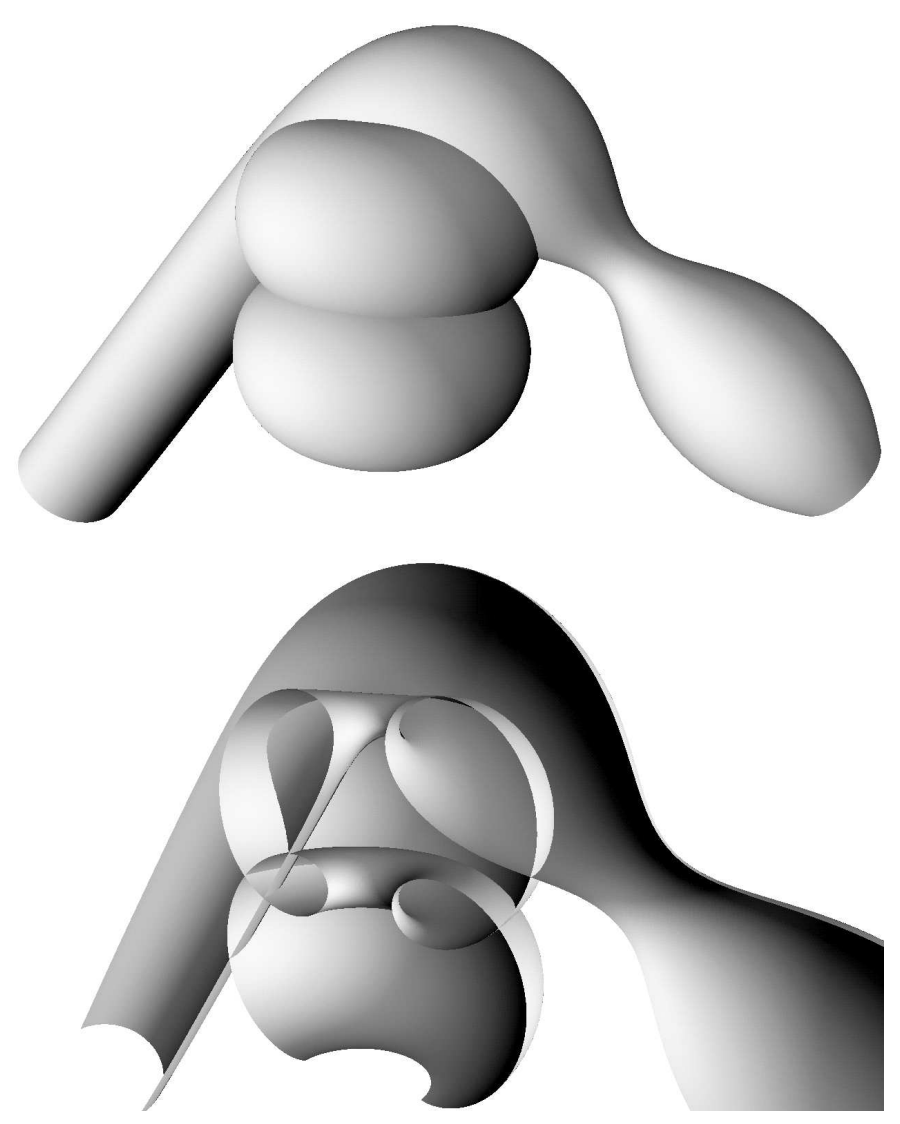

FIGURE 11. Views of an asymmetric 3-noid whose ends are asymptotically a cylinder, a nodoid, and an unduloid. The downward force of cylinder and unduloid are balanced by an upward force of the nodoid.

the Hopf differential does not see the cone point. The residue at each cone point $p$ is chosen to be $\pm A_{\lambda}(0, b, c)$ with $b^{2}+c^{2}=0$ so that the monodromy around $p$ is $-I$ and the surface closes at $p$ for any initial condition.

\section{D. Experimental Evidence}

That the meromorphic potentials in the DPW initial value problem $(2-1)$ for these examples are locally those of Delaunay surfaces is not sufficient to guarantee global existence: initial conditions must also be found which simultaneously close all the ends. While it it yet unproved that such initial conditions exist, these are conjectured on the basis of computational evidence: we have computed approximate initial conditions with a minimizing algorithm.

This computational algorithm implements conjugate gradient methods [Press et al. 1992] to find 
a DPW initial condition $C$ that minimizes, for a fixed potential, an error-measure $\varepsilon(C)$ on the monodromies:

$$
\varepsilon(C)=\sum_{n}\left\|\left.\mathcal{M}_{n}(C)\right|_{\lambda=1}+I\right\|+\left\|\left.\frac{d}{d \lambda} \mathcal{M}_{n}(C)\right|_{\lambda=1}\right\|
$$

$\mathcal{M}_{n}(C)$ is the monodromy of the extended frame around the $n$-th end induced by $C$. The sum is taken over a set of generators of the fundamental group of the underlying Riemann surface. This method was used to create the $k$-noids shown in Figures 9-12.

\section{E. Further Directions}

Recent experiments give evidence for other genuszero $k$-noids: trinoids with three nodoid ends, families of $k$-noids with $k>3$, and 4-noids with noncoplanar end axes.

A monodromy-closer under development finds initial conditions more efficiently by unitarizing the monodromies simultaneously.

\section{ACKNOWLEDGMENTS}

We are grateful to Franz Pedit and Josef Dorfmeister for helpful discussions, and to Rob Kusner for his encouragement.

This work was partially supported by NSF grants DMS-9626804 and DMS-9705479. Kilian was also partially supported by SFB 288 at Technische Universität Berlin, and Schmitt by NFS grant DMS9704949.

\section{ELECTRONIC AVAILABILITY}

For further information about CMC surfaces, the DPW construction, and the dpwlab software, visit the site of The Center for Geometry, Analysis, Numerics and Graphics (GANG) at www.gang.umass. edu, or write Schmitt at nick@gang.umass.edu.

\section{REFERENCES}

[Bobenko 1991] A. I. Bobenko, "Constant mean curvature surfaces and integrable equations", Uspekhi Mat.

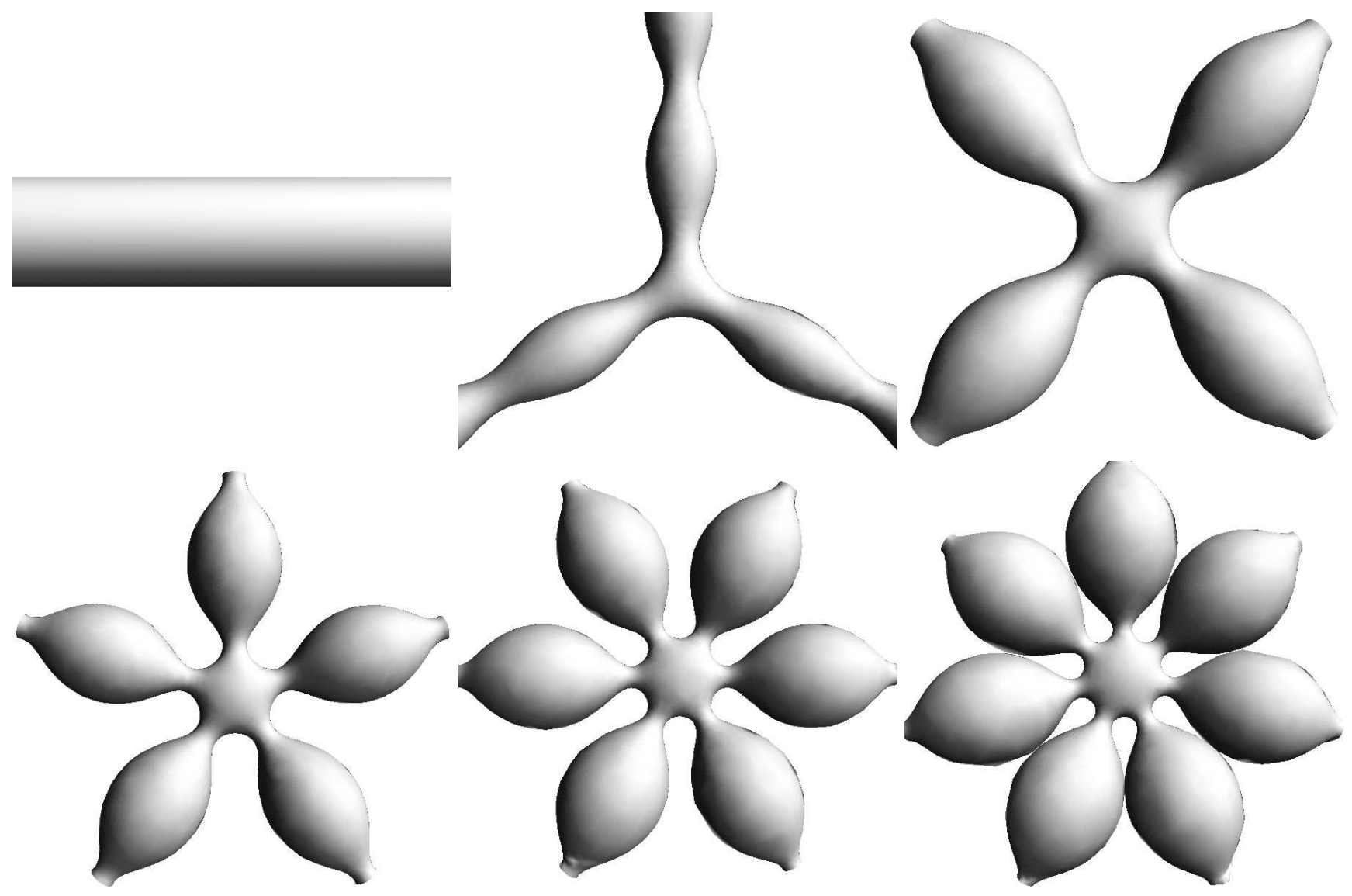

FIGURE 12. Maximal equilateral genus-zero $k$-noids. 
Nauk 46:4 (1991), 3-42, 192. In Russian; translated in Russian Math. Surveys 46:4 (1991), 1-45.

[Bobenko 1994] A. I. Bobenko, "Surfaces in terms of 2 by 2 matrices. Old and new integrable cases", pp. 83-127 in Harmonic maps and integrable systems, edited by A. P. Fordy and J. C. Wood, Aspects of mathematics, E 23, Vieweg, Braunschweig, 1994.

[Burstall and Pedit 1994] F. E. Burstall and F. Pedit, "Harmonic maps via Adler-Kostant-Symes theory", pp. 221-272 in Harmonic maps and integrable systems, edited by A. P. Fordy and J. C. Wood, Aspects of Mathematics E23, Vieweg, Braunschweig, 1994.

[Burstall and Pedit 1995] F. E. Burstall and F. Pedit, "Dressing orbits of harmonic maps", Duke Math. J. 80:2 (1995), 353-382.

[Dorfmeister and Haak $\geq 2000]$ J. Dorfmeister and G. Haak, "Construction of non-simply connected CMC surfaces via dressing". In preparation.

[Dorfmeister and Wu 1993] J. Dorfmeister and H. Y. Wu, "Constant mean curvature surfaces and loop groups", J. Reine Angew. Math. 440 (1993), 43-76.

[Dorfmeister et al. 1998] J. Dorfmeister, F. Pedit, and $\mathrm{H}$. Wu, "Weierstrass type representation of harmonic maps into symmetric spaces", Comm. Anal. Geom. 6:4 (1998), 633-668.

[Große-Brauckmann 1993] K. Große-Brauckmann, "New surfaces of constant mean curvature", Math. Z. 214:4 (1993), 527-565.

[Große-Brauckmann et al. 1999] K. Große-Brauckmann, R. Kusner, and J. Sullivan, "Constant mean curvature surfaces with three ends", Preprint 5.1, University of Massachusetts (GANG), Amherst, 1999. Submitted to Proc. Nat. Acad. Sci. USA.
[Korevaar et al. 1989] N. J. Korevaar, R. Kusner, and B. Solomon, "The structure of complete embedded surfaces with constant mean curvature", J. Differential Geom. 30:2 (1989), 465-503.

[Kusner 1991] R. Kusner, "Bubbles, conservation laws, and balanced diagrams", pp. 103-108 in Geometric analysis and computer graphics (Berkeley, 1988), edited by P. Concus et al., Math. Sci. Res. Inst. Publ. 17, Springer, New York, 1991.

[Lerner and Sterling 1995] D. Lerner and I. Sterling, "Construction of constant mean curvature surfaces using the Dorfmeister-Pedit-Wu representation of harmonic maps", in Proceedings of the Symposium on Differential Geometry, Hamiltonian Systems, and Operator Theory (Mona, Jamaica, 1994), The University Printers (Univ. of the West Indies), 1995.

[Press et al. 1992] W. H. Press, S. A. Teukolsky, W. T. Vetterling, and B. P. Flannery, Numerical recipes in $C, 2$ nd ed., Cambridge University Press, Cambridge, 1992.

[Pressley and Segal 1986] A. Pressley and G. Segal, Loop groups, The Clarendon Press Oxford University Press, New York, 1986.

[Smyth 1993] B. Smyth, "A generalization of a theorem of Delaunay on constant mean curvature surfaces", pp. 123-130 in Statistical thermodynamics and differential geometry of microstructured materials (Minneapolis, MN, 1991), edited by H. T. Davis and J. C. C. Nitsche, IMA Vol. Math. Appl. 51, Springer, New York, 1993.

[Sterling and Wente 1993] I. Sterling and H. C. Wente, "Existence and classification of constant mean curvature multibubbletons of finite and infinite type", Indiana Univ. Math. J. 42:4 (1993), 1239-1266.

[Timmreck et al. 1994] M. Timmreck, U. Pinkall, and D. Ferus, "Constant mean curvature planes with inner rotational symmetry in Euclidean 3-space", Math. Z. 215:4 (1994), 561-568.

Martin Kilian, Center for Geometry, Analysis, Numerics and Graphics, Deptartment of Mathematics, University of Massachusetts, Amherst, MA 01003, United States (kilian@gang.umass.edu)

Ian McIntosh, Department of Mathematics, University of York, York YO10 5DD, United Kingdom (im7@york.ac.uk)

Nicholas Schmitt, Center for Geometry, Analysis, Numerics and Graphics, Deptartment of Mathematics, University of Massachusetts, Amherst, MA 01003, United States (nick@math.umass.edu) 